measuring HIV-RNA copies (Q-PCR: HIV Monitor, Hoffmann-La Roche, Milan, Italy) in the plasma of the patients collected concomitantly to the lymph nodal biopsy, and the values were referred to $1 \mathrm{ml}$ of plasma.

Results from these eight patients are shown in the table. There is a strong correlation $(r=0.97 ; P<0.01)$ between the number of the HIV-RNA copies in the plasma and the number of lymphoid infected cells in the lymph nodes. All lymph nodes showed histological HIV-related damage ranging from follicular hyperplasia to follicular involution; no patient showed follicular depletion.

To our knowledge this is the first report showing, a positive correlation between the extent of plasma viremia and viral load in the lymphoid tissue.

F. DIANZANI ${ }^{1}$, G. ANTONELli' ${ }^{2}$, E. RIVA ${ }^{1}$, S. UCCINI ${ }^{3} \&$ G. VISCO ${ }^{4}$ 'Institute of Virology

University "La Sapienza", Rome, Italy

${ }^{2}$ Department of Biomedicine

University of Pisa, Pisa, Italy

\author{
${ }^{3}$ Department of Experimental \\ Medicine and Pathology \\ University "La Sapienza," Rome, Italy \\ "II Division of Infectious Diseases \\ Hospital "L. Spallanzani" \\ Rome, Italy
}

1. Pantaleo, G. et al. HIV infection is active and progressive in lymphoid tissue during the clinically latent stage of disease. Nature 362, 355-358 (1993).

3. Dianzani, F. et al. Zidovudine induces the expression of cellular resistance affecting its antiviral activity. AIDS Res. Hum. Retrovir. 10, 1471-1477 (1994).

\title{
A closer look at malaria in India
}

To the editor - There are several errors in your News article "Malaria drug import raises fears of resistance" (Nature Medicine 2, 496; 1996). It is stated that there are only two drugs available today for use against drug-resistant Plasmodium falciparum. Artemisinin was overlooked. More than 200 articles have been published on this drug, the most effective available today for treating drug-resistant Plasmodium falciparum infection. Use of artemisinin in Viet Nam has reduced considerably the morbidity and mortality caused by malaria in that country. Our own trials in India have demonstrated its efficacy, and we are keeping it in reserve. The statement that "there is no real urgency" is not correct; there is great urgency given the enormity of the problem. Finally, the name of the Drugs Controller of India is P. Das Gupta, not P.R. Das Gupta, who was the former Director of the National AIDS Control Programme.

\section{RANJIT ROY CHAUDHURY}

Department of Health

New Delhi, India 110067

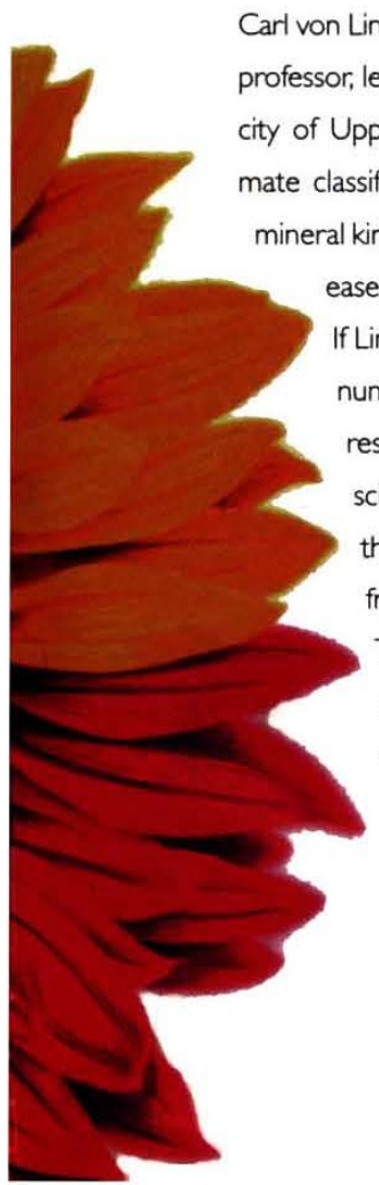

Carl von Linné: 18th century botanist, researcher, physician, professor, lecturer and a resident of the Swedish university city of Uppsala (pronounced OOP-SA-LA). A consummate classifier, Linné systematized the plant, animal and mineral kingdoms as well as drew up a treatise on the diseases known in his day.

If Linné were alive today, he'd be proud of the vast number of diverse and important scientific fields researchers are involved in. Our job is to help life scientists find solutions by getting involved in their activities. We're Pharmacia Biotech-also from Uppsala.

The diversity of our involvement makes us a little tricky to classify at times. After all, our specialists work with such disciplines as (get ready): cell separation, cell culture, nucleic acid purification. PCR-related areas, cDNA synthesis and cloning, vectors, nucleic acid electrophoresis, hybridization, sequencing, gene expression, restriction enzymes, modifying enzymes, nucleotides, oligonucleotides, polynucleotides, oligonucleotide synthesis, chromatography media, reagents, BioProcess chromatography, electrophoresis systems, electrophoresis media and spectrophotometry. As you can see, our company works with more than just one scientific discipline.

\section{Do you?}

If so, give us a call: I (800) 526-3593 in the USA; +81 334926949 in Japan; or +46 18165000 in Europe and the rest of the world. Or visit us on the Internet: http://muw. biotech.pharmacia.se.

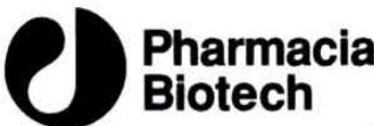

Uppsala. Sweden. (And the rest of the world) 